PROCEEDINGS OF THE

AMERICAN MATHEMATICAL SOCIETY

Volume 132, Number 9, Pages 2703-2712

S 0002-9939(04)07431-3

Article electronically published on April 21, 2004

\title{
SUB-EXPONENTIAL DECAY OF OPERATOR KERNELS FOR FUNCTIONS OF GENERALIZED SCHRÖDINGER OPERATORS
}

\author{
JEAN-MARC BOUCLET, FRANÇOIS GERMINET, AND ABEL KLEIN
}

(Communicated by Joseph A. Ball)

\begin{abstract}
We study the decay at large distances of operator kernels of functions of generalized Schrödinger operators. We prove sub-exponential decay for functions in Gevrey classes and exponential decay for real analytic functions.
\end{abstract}

\section{INTRODUCTION AND RESULT}

In a previous paper two of the authors proved that operator kernels of smooth functions of generalized Schrödinger operators decay faster than any polynomial GK2. The operator kernel of a bounded operator $A$ acting on $\mathrm{L}^{2}\left(\mathbb{R}^{d}, \mathrm{~d} q ; \mathbb{C}^{k}\right)$ is defined as $\chi_{x} A \chi_{y}$, where

$$
\chi_{x}(q)=\chi_{0}(q-x), \quad q, x \in \mathbb{R}^{d},
$$

and $\chi_{0}$ is the characteristic function of the cube centered at 0 with side 1 . We use the standard notation $\langle x\rangle=\left(1+|x|^{2}\right)^{1 / 2}$ for $x \in \mathbb{R}^{d}$. Given a generalized Schrödinger operator $H$ (see [GK2] and Remark 1.3] below) and a $C^{\infty}$ function $f$ with sufficiently fast decay at $\infty$, namely,

$$
\int_{\mathbb{R}}\left|f^{(k)}(u)\right|\langle u\rangle^{k-1} \mathrm{~d} u<\infty \text { for } k=0,1,2, \ldots,
$$

it is shown in GK2, Theorem 2] that for all $n=0,1,2, \ldots$ one has

$$
\left\|\chi_{x} f(H) \chi_{y}\right\| \leq C_{n, f}\langle x-y\rangle^{-n}, \quad x, y \in \mathbb{R}^{d}
$$

(with a control on $C_{n, f}$ in terms of integrals as in (2)). Such kernel decay estimates are of interest from a purely mathematical point of view (see [Si, Da1, Da2]). Moreover, controls like (3) have already been quite useful in applications, e.g., GK3, GK4, ElGr, Tc.

The purpose of this paper is to improve on (3) and show that if the $C^{\infty}$ function $f$ belongs to a Gevrey-like class (see Definition 1.1), then

$$
\left\|\chi_{x} f(H) \chi_{y}\right\| \leq C \mathrm{e}^{-c|x-y|^{\alpha}}, \quad x, y \in \mathbb{R}^{d},
$$

for some $\alpha \in(0,1]$ depending on $f$. This extension was motivated by some applications which are discussed at the end of this section.

Received by the editors February 13, 2003 and, in revised form, July 7, 2003.

2000 Mathematics Subject Classification. Primary 81Q10, 47F05; Secondary 35P05.

Key words and phrases. Schrödinger operator, acoustic operator, Maxwell operator, CombesThomas estimate, operator kernel, Gevrey class.

The third author was supported in part by NSF Grant DMS-0200710. 
We recall that a function $f$ belongs to the Gevrey class of order $a \geq 1$, denoted by $G^{a}(\mathbb{R})$ (e.g., $[\mathrm{Ho}]$ ), if $f \in C^{\infty}(\mathbb{R})$ and for each compact subset $K \subset \mathbb{R}$ there exists a constant $C_{K}$ such that

$$
\left|f^{(k)}(u)\right| \leq C_{K}\left(C_{K}(k+1)^{a}\right)^{k}, \quad k=0,1,2, \ldots, \quad u \in K .
$$

Note that being Gevrey is thus a local property. The class $G^{1}(\mathbb{R})$ is the set of real analytic functions, and authors sometimes refer to Gevrey classes only for $a>1$.

We emphasize that for any $a>1$ the Gevrey class $G^{a}(\mathbb{R})$ contains compactly supported functions. (For example, the function $f_{a}(x)=\exp \left(-\left(1-x^{2}\right)^{-\frac{1}{a-1}}\right)$ for $|x|<1$ and $f_{a}(x)=0$ for $|x| \geq 1$ belongs to $G^{a}(\mathbb{R})$.) In fact, most of the smooth cut-off functions that are commonly used can be chosen in $G^{a}(\mathbb{R})$ with $a$ arbitrarily close to 1 . We will denote the class of compactly supported functions in $G^{a}(\mathbb{R})$ by $G_{\text {comp }}^{a}(\mathbb{R})$. Of course many interesting functions are non-compactly supported, especially analytic ones, and we need to consider a larger set of functions.

To allow functions supported either on $\mathbb{R}$ or on a half-line, we combine the local Gevrey estimate (5) with the condition of decay at $\infty$ given by (2), and make the following definition. (By $C_{a, b, \ldots}$ we always denote a constant depending on $a, b, \ldots$.)

Definition 1.1. Let $I$ be an open interval and $a \geq 1$. A function $f \in C^{\infty}(\mathbb{R})$ is $\mathrm{L}^{1}$-Gevrey of class $a$ on $I$ if for all $k=0,1,2, \ldots$ we have

$$
\left\|f^{(k)}\langle u\rangle^{k-1}\right\|_{L^{1}(I)}=\int_{I}\left|f^{(k)}(u)\right|\langle u\rangle^{k-1} \mathrm{~d} u \leq C_{f, I}\left(C_{f, I}(k+1)^{a}\right)^{k} .
$$

As far as the self-adjoint operator $H$ is concerned, the sole property we shall require is that a suitable Combes-Thomas estimate CoTh GK2 holds. We state it as a definition.

Definition 1.2. The self-adjoint operator $H$ on $\mathrm{L}^{2}\left(\mathbb{R}^{d}, \mathrm{~d} q ; \mathbb{C}^{k}\right)$ has property $(C T)$ if there exists a constant $C$ (depending on $H, d$ and $k$ ) such that for all $z \notin \sigma(H)$ and all $x, y \in \mathbb{R}^{d}$ we have

$$
\left\|\chi_{x}(H-z)^{-1} \chi_{y}\right\| \leq \frac{C}{\eta_{z}} \exp \left(-\frac{C \eta_{z}}{1+\eta_{z}+|z|}|x-y|\right)
$$

where $\eta_{z}=\mathrm{d}(z, \sigma(H))$.

Remark 1.3. The inequality (7) is proven in [GK2, Corollary 1] for generalized Schrödinger operators, a class of semibounded second-order partial differential operators of mathematical physics which includes the Schrödinger operator, magnetic Schrödinger operator, and the classical wave operators (i.e., acoustic operator, Maxwell operator, and other second-order partial differential operators associated with classical wave equations). Specific examples of operators with property (CT) are given by the Schrödinger operator

$$
H=-\Delta+V^{(1)}+V^{(2)} \text { on } \mathrm{L}^{2}\left(\mathbb{R}^{d}, \mathrm{~d} x\right),
$$

with $0 \leq V^{(1)} \in \mathrm{L}_{\text {loc }}^{1}$ and $V^{(2)}$ relatively form bounded with respect to $-\Delta$ with relative bound $<1$, the acoustic operator

$$
A=-\frac{1}{\sqrt{\kappa}} \nabla \cdot \frac{1}{\rho} \nabla \frac{1}{\sqrt{\kappa}} \text { on } \mathrm{L}^{2}\left(\mathbb{R}^{d}, \mathrm{~d} x\right),
$$


where $\kappa(x)$ is the compressibility and $\varrho(x)$ is the mass density, and the Maxwell operator

$$
M=\frac{1}{\sqrt{\mu}} \nabla \times \frac{1}{\varepsilon} \nabla \times \frac{1}{\sqrt{\mu}} \text { on } \mathrm{L}^{2}\left(\mathbb{R}^{3}, \mathrm{~d} x ; \mathbb{C}^{3}\right),
$$

where $\mu(x)$ is the magnetic permeability and $\varepsilon(x)$ is the dielectric constant, with $\kappa(x), \varrho(x), \mu(x), \varepsilon(x)$ bounded, uniformly strictly positive, measurable functions.

Our results are stated in the following theorem.

Theorem 1.4. Let $H$ be a self-adjoint operator on $\mathrm{L}^{2}\left(\mathbb{R}^{d}, \mathrm{~d} q ; \mathbb{C}^{k}\right)$ with property $(C T), I$ an open interval containing the spectrum of $H$. Then, given a real number $a \geq 1$, we have:

(i) If $a>1$, then for each $a^{\prime}>a$ there exists $C_{I, a, a^{\prime}}>0$, such that for all $\mathrm{L}^{1}$-Gevrey functions $f$ of class a on $I$,

$$
\left\|\chi_{x} f(H) \chi_{y}\right\| \leq C_{I, a, a^{\prime}}\left(\mathrm{e} C_{f, I}\right)^{3+|x-y|^{\frac{1}{a^{\prime}}}} \exp \left(-\frac{a^{\prime}-a}{4 a^{\prime}}|x-y|^{\frac{1}{a^{\prime}}} \ln |x-y|\right)
$$

for all $x, y \in \mathbb{R}^{d}$. As a consequence, for each $a^{\prime}>a$, there exist constants $C_{f, I, a, a^{\prime}}>0$ and $c_{a, a^{\prime}}>0$ such that

$$
\left\|\chi_{x} f(H) \chi_{y}\right\| \leq C_{f, I, a, a^{\prime}} \exp \left(-c_{a, a^{\prime}}|x-y|^{\frac{1}{a^{r}}}\right) \text { for all } x, y \in \mathbb{R}^{d} \text {. }
$$

(ii) If $a=1$, then there exist constants $C_{I}, c_{I}>0$ such that for all $\mathrm{L}^{1}$-Gevrey functions $f$ of class a on $I$,

$$
\left\|\chi_{x} f(H) \chi_{y}\right\| \leq C_{I} \frac{C_{f, I}\langle x-y\rangle}{1+\ln C_{f, I}} \exp \left(-\frac{c_{I}}{C_{f, I}\left(1+\ln C_{f, I}\right)}|x-y|\right)
$$

for all $x, y \in \mathbb{R}^{d}$.

Remark 1.5. The mass $\frac{a^{\prime}-a}{4 a^{\prime}}$ in (11) can be chosen arbitrarily close to $\frac{a^{\prime}-a}{a^{\prime}}$. This is done by modifying the proof by choosing $1-s=\frac{1+\gamma\left(a^{\prime}-a\right)}{a^{\prime}}$ in (42) with $\gamma$ close to zero.

Remark 1.6. If $f$ has an analytic continuation to a region where one can use a suitable contour, then (13) is a rather simple consequence of the Combes-Thomas estimate and the Cauchy integral formula. In our theorem, no condition on the form of the domain of analyticity of $f$ in $\mathbb{C}$ is assumed. We nevertheless obtain exponential decay in $|x-y|$.

To discuss the range of application of Theorem 1.4 and more precisely of condition (6), we introduce the weighted Gevrey class $G_{\gamma}^{a}(I)$ in the following way: given $\gamma \in \mathbb{R}, a \geq 1$, and an open interval $I$, we say that $f \in G_{\gamma}^{a}(I)$ if $f \in C^{\infty}$ and there exists $C \geq 0$ such that

$$
\sup _{u \in I}\left|f^{(k)}(u)\langle u\rangle^{k+\gamma}\right| \leq C\left(C(k+1)^{a}\right)^{k}, \quad k=0,1,2, \ldots
$$

The class $G_{\gamma}^{a}(I)$ is never trivial (i.e., reduced to 0 ): if $a>1$ it contains all functions of $G_{\text {comp }}^{a}(\mathbb{R})$ that are supported in $I$, and if $a=1$ it can be easily seen that for $t>0$ and $z \notin I$, the functions

$$
\exp \left(-t u^{2}\right), \quad \exp (-t u), \quad \exp (-i u t) \exp \left(-u^{2}\right), \quad \frac{1}{u-z}
$$


belong to $G_{\gamma}^{1}((\lambda,+\infty))$ for all $\lambda$ and all $\gamma$ for the first three, and to $G_{1}^{1}(\mathbb{R})$ for the last one.

Functions in $G_{\gamma}^{a}(I), \gamma>0$, clearly satisfy (6). Moreover, if $f_{1} \in G_{\gamma_{1}}^{a_{1}}(I)$ and $f_{2} \in G_{\gamma_{2}}^{a_{2}}(I), \gamma_{1}, \gamma_{2} \in \mathbb{R}, a_{1}, a_{2} \geq 1$, then one can verify that

$$
f_{1} f_{2} \in G_{\gamma}^{a}(I), \quad a=\max \left(a_{1}, a_{2}\right), \quad \gamma=\gamma_{1}+\gamma_{2} .
$$

This is of interest since in applications we often have to consider cutoffs of the resolvent (see for instance [GK3]), that is, $f(H)$ with

$$
f(u)=\left(1-f_{1}(u)\right) \frac{1}{u-\lambda_{0}}, \quad f_{1} \in C_{\text {comp }}^{\infty}(\mathbb{R}), \quad f_{1} \equiv 1 \text { near } \lambda_{0} \in \mathbb{R} .
$$

By choosing $f_{1} \in G_{\text {comp }}^{a}(\mathbb{R})$ with $a$ arbitrarily close to 1 , we can deduce from our remarks that $f \in G_{1}^{a}(\mathbb{R})$, and hence get the sub-exponential decay of $\left\|\chi_{x} f(H) \chi_{y}\right\|$ by (12).

We end this section by discussing some applications of Theorem 1.4 to the theory of Schrödinger operators:

(1) In the context of random Schrödinger operators, Theorem 1.4 can be used to extend the results of GK3 to models where only a Wegner estimate with sub-exponential controls is available, as in the case of Bernoulli-Anderson models and polymers [CKM] KLS, DBG, DSS, JSBS]. In particular, it allows the definition of the strong insulator region in these cases, similar to what is done in GK3 for models with a Wegner estimate with polynomial controls.

(2) Still in the context of random Schrödinger operators, Theorem 1.4, combined with [GK1, Theorem 3.8], provides a simple and quick proof of subexponential decay of any order of the operator kernel of the Fermi projector $P_{E_{F}}=\chi_{\left(-\infty, E_{F}\right]}(H)$, provided the Fermi energy $E_{F}$ lies inside the strong insulator region (equivalently, provided $E_{F}$ lies in the region of applicability of the multiscale analysis).

(3) In GKT, an approximation lemma shows that the weight given to balls of radius $\varepsilon$ by spectral measures of Hamiltonians that coincide on a region of size $\mathcal{O}\left(\varepsilon^{-1}\right)$ are close up to a polynomial term in $\varepsilon$. Combining the methods of the present paper with the ones of [GKT] improves the latter by showing that the error term is actually sub-exponentially small. It moreover allows one to treat more general potentials, since they could be assumed to be sub-exponentially bounded rather than polynomially.

(4) We note that if $f$ is a real analytic function, then it follows from (13) that the moments $\left\|\langle X\rangle^{p / 2} \mathrm{e}^{-i H t} f(H) \chi_{0}\right\|^{2}$ cannot grow faster than $\left(t(\ln t)^{3}\right)^{p}$. (This can be seen by cutting $\mathbb{R}^{d}$ into two regions: $F(|x|) \leq t \ln t$ and $F(|x|) \geq t \ln t$, where $F(u)=u(\ln u)^{-2}$.)

\section{ProOF}

In order to use the Combes-Thomas estimate (7), we need to express $f(H)$ in terms of the resolvent $R(z)=(H-z)^{-1}$. This is done by the Helffer-Sjöstrand formula $\mathrm{HeSj}$, DiSj]

$$
f(H)=\frac{1}{\pi} \iint \bar{\partial} \tilde{f}(u+i v) R(u+i v) \mathrm{d} u \mathrm{~d} v, \quad \bar{\partial}=\frac{1}{2}\left(\partial_{u}+i \partial_{v}\right),
$$


where $\tilde{f}$ is a quasi-analytic extension of $f$, i.e., $\bar{\partial} \tilde{f}=\mathcal{O}(v)$ near $v=0$, with conditions ensuring the convergence of the integral. Here we use an extension $\tilde{f}$ of $f$ similar to the one in [GK2], with one difference: we add a smooth cutoff $\varphi(u)$ around the spectrum of $H$ (we can thus allow for a larger class of functions $f$, such as $\mathrm{e}^{-x}$ if the operator $H$ is bounded from below):

$$
\begin{aligned}
& \tilde{f}_{n}(u+i v)=\varphi(u) \tau(v /\langle u\rangle) S_{n} \tilde{f}(u+i v), \\
& \text { with } \quad S_{n} \tilde{f}(u+i v)=\sum_{k=0}^{n} \frac{1}{k !} f^{(k)}(u)(i v)^{k},
\end{aligned}
$$

where the function $\tau$ is smooth such that $\tau(t)=1$ for $|t| \leq 1 / 2$ and $\tau(t)=0$ for $|t| \geq 1$; the function $\varphi$ is smooth as well and satisfies $\varphi(u)=1$ near $\sigma(H)$, and $\operatorname{supp} \varphi \subset I$.

In all that follows, we set $\rho(u, v)=\varphi(u) \tau(v /\langle u\rangle)$. Then we have

$$
\bar{\partial} \tilde{f}_{n}(u+i v)=\bar{\partial} \rho(u, v) \sum_{k=0}^{n} \frac{1}{k !} f^{(k)}(u)(i v)^{k}+\frac{\rho(u, v)}{2} \frac{1}{n !} f^{(n+1)}(u)(i v)^{n} .
$$

Note that we have

$$
\bar{\partial} \tau(v /\langle u\rangle)=\frac{1}{2\langle u\rangle}\left(-\frac{u}{\langle u\rangle} \frac{v}{\langle u\rangle} \tau^{\prime}(v /\langle u\rangle)+i \tau^{\prime}(v /\langle u\rangle)\right) .
$$

The main idea of the proof is to write

$$
\begin{aligned}
f(H)= & \frac{1}{\pi} \iint_{|v|<\epsilon\langle u\rangle} \bar{\partial} \tilde{f}_{n}(u+i v) R(u+i v) \mathrm{d} u \mathrm{~d} v \\
& \quad+\frac{1}{\pi} \iint_{|v| \geq \epsilon\langle u\rangle} \bar{\partial} \tilde{f}_{n}(u+i v) R(u+i v) \mathrm{d} u \mathrm{~d} v \\
= & \mathrm{I}+\mathrm{II},
\end{aligned}
$$

with appropriate choices of $\epsilon$ and $n$.

In all that follows, the constants $C, c$ may depend on $I$ but never on $\epsilon, n, f$ or $x, y$.

Lemma 2.1. There exist $C, c>0$ such that

$$
\begin{aligned}
& \| \chi_{x} \text { I } \chi_{y} \| \\
& \quad \leq C \mathrm{e}^{-c|x-y|} \sum_{k=0}^{n} \frac{1}{k !}\left\|f^{(k)}\langle u\rangle^{k-1}\right\|_{L^{1}(I)}+\frac{C \epsilon^{n}}{n !}\left\|f^{(n+1)}\langle u\rangle^{n}\right\|_{\mathrm{L}^{1}(I)}
\end{aligned}
$$

for all $n \geq 1,0<\epsilon \leq 1 / 2$ and $x, y \in \mathbb{R}^{d}$. The constant $c$ in the exponent depends only on the choice of $\varphi$ (through its support and the distance of its support to the open interval I).

Proof. Assuming that $\epsilon \leq 1 / 2$ implies that the term involving $\tau^{\prime}$ in (19) does not contribute. Actually we only have to consider $\varphi^{\prime}(u) \tau(v /\langle u\rangle) S_{n} \tilde{f}$ and the last term of (19). We first estimate the contribution of the last term by using the basic 
estimate $\|R(u+i v)\| \leq|v|^{-1}$, and we get

$$
\begin{gathered}
\left\|\iint_{|v|<\epsilon\langle u\rangle} \frac{\rho(u, v)}{n !} f^{(n+1)}(u)(i v)^{n} R(u+i v) \mathrm{d} u \mathrm{~d} v\right\| \\
\leq \frac{C}{n !} \int_{I} \int_{-\epsilon\langle u\rangle}^{\epsilon\langle u\rangle}\left|f^{(n+1)}(u)\right||v|^{n-1} \mathrm{~d} v \mathrm{~d} u \\
\leq \frac{2 C}{n !} \int_{I}\left|f^{(n+1)}(u)\right|(\epsilon\langle u\rangle)^{n} \mathrm{~d} u \\
\leq \frac{2 C}{n !} \epsilon^{n}\left\|f^{(n+1)}\langle u\rangle^{n}\right\|_{L^{1}(I)} .
\end{gathered}
$$

Note that we have not used the Combes-Thomas estimate for this part. Next we consider the term involving $\varphi^{\prime}(u)$. This term is compactly supported outside $\sigma(H)$ with respect to $z=u+i v$, so that $\eta_{z}=\operatorname{dist}(z, \sigma(H))$ is uniformly bounded away from 0 and $\langle u\rangle$ is bounded. The Combes-Thomas estimate (7) implies that

$$
\begin{gathered}
\left\|\iint_{\frac{|v|}{\langle u\rangle}<\epsilon} \varphi^{\prime}(u) \tau(v /\langle u\rangle) S_{n} \tilde{f}(u+i v) \chi_{x} R(u+i v) \chi_{y} \mathrm{~d} u \mathrm{~d} v\right\| \\
\leq C \mathrm{e}^{-c|x-y|} \int_{\operatorname{supp} \varphi^{\prime}} \int_{-\epsilon\langle u\rangle}^{\epsilon\langle u\rangle} \sum_{k=0}^{n} \frac{1}{k !}\left|f^{(k)}(u)\right||v|^{k} \mathrm{~d} v \mathrm{~d} u \\
\leq C \mathrm{e}^{-c|x-y|} \sum_{k=0}^{n} \frac{1}{k !}\left\|f^{(k)}\langle u\rangle^{k-1}\right\|_{L^{1}(I)},
\end{gathered}
$$

where we used the fact that $\varphi^{\prime}$ is compactly supported and $\epsilon \leq 1 / 2$.

We consider now the estimates for II.

Lemma 2.2. There exist $C, c>0$ such that

$$
\begin{aligned}
& \| \chi_{x} \text { II } \chi_{y} \| \\
& \quad \leq C \mathrm{e}^{-c|x-y|} \sum_{k=0}^{n} \frac{1}{k !}\left\|f^{(k)}\langle u\rangle^{k-1}\right\|_{\mathrm{L}^{1}(I)}+\frac{C}{n !} \mathrm{e}^{-c \epsilon|x-y|}\left\|f^{(n+1)}\langle u\rangle^{n}\right\|_{\mathrm{L}^{1}(I)},
\end{aligned}
$$

for all $\epsilon>0, n \geq 1, x, y \in \mathbb{R}^{d}$.

Proof. On the domain of integration that we are considering, one has $|v| \leq \eta=$ $\operatorname{dist}(u+i v, \sigma(H))$ and $\epsilon\langle u\rangle \leq|v| \leq\langle u\rangle$. Thus the Combes-Thomas estimate (7) reduces to

$$
\left\|\chi_{x} R(u+i v) \chi_{y}\right\| \leq \frac{C}{|v|} \mathrm{e}^{-c \frac{|v|}{\langle u\rangle}|x-y|} \leq \frac{C}{|v|} \mathrm{e}^{-c \epsilon|x-y|},
$$

for all $x, y \in \mathbb{R}^{d}$, with $C, c>0$ constants independent of $x, y, u, v$. To evaluate $\| \chi_{x}$ II $\chi_{y} \|$ we use (19) and treat separately the sum and the last term. We use (29) only for the latter, getting

$$
\begin{aligned}
\| \chi_{x} \text { II } \chi_{y} \| \leq & \frac{1}{\pi} \iint|\bar{\partial} \rho(u, v)|\left\{\sum_{k=0}^{n} \frac{1}{k !}\left|f^{(k)}(u)\right||v|^{k}\right\}\left\|\chi_{x} R(u+i v) \chi_{y}\right\| \mathrm{d} v \mathrm{~d} u \\
& +C \mathrm{e}^{-c \epsilon|x-y|} \int_{I} \int_{-\langle u\rangle}^{\langle u\rangle}\left\{\frac{1}{n !}\left|f^{(n+1)}(u)\right||v|^{n-1}\right\} \mathrm{d} v \mathrm{~d} u .
\end{aligned}
$$


The last term clearly yields the second term in (28).

Now the term $|\bar{\partial} \rho|=\left|\varphi^{\prime} \tau+\varphi \bar{\partial} \tau\right|$ will be yielding a factor $1 /\langle u\rangle$, coming either from (201) or from the fact that $\varphi^{\prime}$ is compactly supported. In other terms, we will use the bound

$$
|\bar{\partial} \rho(u, v)| \leq \frac{C}{\langle u\rangle} .
$$

Moreover, on the support of $\bar{\partial} \rho$ the distance to the spectrum of $H$ is uniformly bounded away from zero. Indeed, on the support of $\varphi^{\prime}$, a compact set disjoint from the spectrum of $H$, we have $\eta=\operatorname{dist}(u+i v, \sigma(H)) \geq C\langle u\rangle$ for some constant $C>0$. On the support of $\bar{\partial} \tau$ we have $\eta \geq|v| \geq\langle u\rangle / 2$. As a consequence, it follows by (7) that on the support of $\bar{\partial} \rho$ we have

$$
\left\|\chi_{x} R(u+i v) \chi_{y}\right\| \leq \frac{C}{\langle u\rangle} \mathrm{e}^{-c|x-y|} .
$$

Plugging (31) and (32) into the first integral on the right-hand side of (30) leads to

$$
\begin{aligned}
& \iint|\bar{\partial} \rho(u, v)|\left\{\sum_{k=0}^{n} \frac{1}{k !}\left|f^{(k)}(u)\right||v|^{k}\right\}\left\|\chi_{x} R(u+i v) \chi_{y}\right\| \mathrm{d} v \mathrm{~d} u \\
& \leq C \mathrm{e}^{-c|x-y|} \int_{I} \int_{-\langle u\rangle}^{\langle u\rangle} \frac{1}{\langle u\rangle^{2}}\left\{\sum_{k=0}^{n} \frac{1}{k !}\left|f^{(k)}(u)\right||v|^{k}\right\} \mathrm{d} v \mathrm{~d} u \\
& \leq C \mathrm{e}^{-c|x-y|} \sum_{k=0}^{n} \frac{1}{k !}\left\|f^{(k)}\langle u\rangle^{k-1}\right\|_{\mathrm{L}^{1}(I)},
\end{aligned}
$$

giving the first term in (28).

The next lemma gives an estimate on the following quantity, which appears in the last two lemmas, after controlling the norms by ([6):

$$
A_{n, a}=\sum_{k=0}^{n} \frac{1}{k !} C_{f, I}\left(C_{f, I}(k+1)^{a}\right)^{k}+\frac{1}{n !} C_{f, I}\left(C_{f, I}(n+2)^{a}\right)^{(n+1)} .
$$

It will motivate the choice of $\epsilon$ and $n$ in the proof of Theorem 1.4

Lemma 2.3. For all $a \geq 1$, one has

$$
A_{n, a} \leq 3 \mathrm{e}^{4 a} n^{a}\left(\mathrm{e} C_{f, I}\right)^{n+2} n^{(a-1) n}, \quad \forall n \geq 1 .
$$

Proof. First note that $k ! \geq(k / \mathrm{e})^{k}$ for $k \geq 1$, and estimate $\mathrm{e}^{k} C_{f, I}^{k}$ by $\left(\mathrm{e} C_{f, I}\right)^{n}$ for $k \leq n$ (recall that $C_{f, I} \geq 1$ ). Moreover, we have $(k+1)^{a k} / k^{k} \leq \mathrm{e}^{a} k^{(a-1) k}$ and, recalling that $a \geq 1$, we can therefore estimate each term of the sum by the one for $k=n$. In a similar way, $(n+2)^{a(n+1)} / n^{n} \leq \mathrm{e}^{4 a} n^{a(n+1)-n}$. The result now follows easily.

Proof of Theorem 1.4 The last three lemmas show clearly that we have to consider, with $K_{f, I}=\mathrm{e} C_{f, I}$,

$$
\begin{aligned}
& n^{a}\left(K_{f, I}\right)^{n+2} \exp ((a-1) n \ln n-c|x-y|), \\
& n^{a}\left(K_{f, I}\right)^{n+2} \exp ((a-1) n \ln n+n \ln \epsilon), \\
& n^{a}\left(K_{f, I}\right)^{n+2} \exp ((a-1) n \ln n-c \epsilon|x-y|) .
\end{aligned}
$$


To simplify, and with no loss of generality, we may assume that the constants $c$ appearing in (36) and (38) are the same. In both cases $a=1$ and $a>1$, we will choose $n=\mathcal{O}\left(|x-y|^{a}\right)$; thus, if we can show that the exponential terms listed above have the decay announced in the theorem, we shall get the result.

Proof in the case $a=1$. We choose $n \sim \delta|x-y|$ with $\delta>0$ small enough and a fixed $\epsilon>0$ small enough as well. Then (37) gives the following first condition:

$$
\epsilon K_{f, I}<1 \text {. }
$$

Now, by choosing $n$ such that $\delta|x-y|-1 \leq n+2<\delta|x-y|$, (38) yields the second condition:

$$
\delta \ln K_{f, I}-c \epsilon<0 .
$$

Similarly, we obtain the third condition from (36):

$$
\delta \ln K_{f, I}-c<0 .
$$

The existence of $\epsilon, \delta>0$ such that these three conditions are satisfied is clear since we may first choose $\epsilon<1$ satisfying (39) and then $\delta$ small enough satisfying (40), and thus (41) as well. We may choose $\epsilon$ such that $\epsilon K_{f, I}=\mathrm{e}^{-1}$ and $\delta=$ $(c \epsilon) /\left(2 \ln K_{f, I}\right)$. Since $K_{f, I} \geq$ e, each term (36), (37), (38) is bounded by the bound of (37), namely $n K_{f, I}^{2} \exp (-n)$. The estimate (13) follows.

Proof in the case $a>1$. In this part $n$ and $\epsilon$ will both depend on $|x-y|$. Pick $a^{\prime}>a$ as in the theorem. Precisely, we write $\nu=a^{\prime}-a>0$, and set

$$
\begin{array}{r}
\epsilon=|x-y|^{-s}, \quad|x-y|^{\delta} \leq n<|x-y|^{\delta}+1, \\
\text { with } \quad \delta=\frac{1}{a^{\prime}}, \quad 1-s=\frac{1+\frac{1}{2} \nu}{a+\nu} .
\end{array}
$$

Note that $|x-y| \sim n^{1 / \delta}$ and $\epsilon \leq n^{-s / \delta}$; moreover, since we consider large $|x-y|$, we may assume that $|x-y| \geq(n / 2)^{1 / \delta}$. We now have, for $n$ large enough (depending only on $s, \delta, a$ ),

$$
\begin{aligned}
(\text { Eq. 366) } & \leq n^{a}\left(K_{f, I}\right)^{n+2} \exp \left(-\frac{c}{2} n^{\frac{1}{\delta}}+(a-1) n \ln n\right) \\
& \leq\left(K_{f, I}\right)^{n+2} \exp \left(-\frac{c}{4} n^{\frac{1}{\delta}}\right), \\
(\text { Eq. 37) } & \leq n^{a}\left(K_{f, I}\right)^{n+2} \exp \left(-\left(\frac{s}{\delta}-a+1\right) n \ln n\right) \\
& \leq\left(K_{f, I}\right)^{n+2} \exp \left(-\frac{1}{2}\left(\frac{s}{\delta}-a+1\right) n \ln n\right), \\
(\text { Eq. 388) } & \leq n^{a}\left(K_{f, I}\right)^{n+2} \exp \left(-\frac{c}{2} n^{\frac{1-s}{\delta}}+(a-1) n \ln n\right) \\
& \leq\left(K_{f, I}\right)^{n+2} \exp \left(-\frac{c}{4} n^{\frac{1-s}{\delta}}\right),
\end{aligned}
$$

provided

$$
\frac{1-s}{\delta}>1 \quad \text { and } \quad \frac{s}{\delta}-a+1>0 .
$$


But (42) ensures the latter, since

$$
\frac{1-s}{\delta}=1+\nu \quad \text { and } \quad \frac{s}{\delta}-a+1=\frac{1}{2} \nu .
$$

Thus the estimate of the theorem depends only on the slowest term (46), and we obtain

$$
\left\|\chi_{x} f(H) \chi_{y}\right\| \leq C_{a, a^{\prime}}\left(K_{f, I}\right)^{n+2} \exp \left(-\frac{a^{\prime}-a}{4} n \ln n\right) .
$$

We get the result since $n \sim|x-y|^{\delta}=|x-y|^{\frac{1}{a^{\top}}}$ and $K_{f, I}=\mathrm{e} C_{f, I}$.

This finishes the proof of Theorem 1.4

\section{ACKNOWLEDGEMENT}

François Germinet would like to thank Didier Robert for useful discussions.

\section{REFERENCES}

[CKM] R. Carmona, A. Klein, and F. Martinelli: Anderson localization for Bernoulli and other singular potentials, Commun. Math. Phys. 108, 41-66 (1987). MR 88f:82027

[CoTh] J. M. Combes and L. Thomas: Asymptotic behavior of eigenfunctions for multi-particle Schrödinger operators, Commun. Math. Phys. 34, 251-270 (1973). MR 52:12611

[DSS] D. Damanik, R. Sims, and G. Stolz: Localization for one-dimensional, continuum, Bernoulli-Anderson models, Duke Math. J. 114, 59-100 (2002). MR 2003h:82048

[Da1] E. B. Davies: Kernel estimates for functions of second order elliptic operators, Quart. J. Math. Oxford Ser. (2) 39, 37-46 (1988). MR 89c:35123

[Da2] E. B. Davies: Heat kernels and spectral theory, Cambridge University Press, Cambridge, 1990. MR 92a:35035

[DBG] S. De Bièvre and F. Germinet: Dynamical localization for the random dimer Schrödinger operator, J. Statist. Phys. 98, 1135-1147 (2000). MR 2000m:82053

[DiSj] M. Dimassi and J. Sjöstrand: Spectral asymptotics in the semi-classical limit, London Math. Soc. Lecture Note Series 268, Cambridge University Press, Cambridge, 1999. MR 2001b:35237

[ElGr] P. Elbau and G. M. Graf: Equality of bulk and edge Hall conductance revisited, Commun. Math. Phys. 229, 415-432 (2002). MR 2003h:81264

[GKT] F. Germinet, A. Kiselev, and S. Tcheremchantsev: Transfer matrices and transport for $1 D$ Schrödinger operators with singular spectrum, Ann. Inst. Fourier, to appear.

[GK1] F. Germinet and A. Klein: Bootstrap multiscale analysis and localization in random media, Commun. Math. Phys. 222, 415-448 (2001). MR 2002m:82035

[GK2] F. Germinet and A. Klein: Operator kernel estimates for functions of generalized Schrödinger operators, Proc. Amer. Math. Soc. 131, 911-920 (2003). MR 2003k:47067

[GK3] F. Germinet and A. Klein: A characterization of the metal-insulator transport transition, submitted.

[GK4] F. Germinet and A. Klein: The Anderson metal-insulator transport transition, to appear in Contemp. Math.

[HeSj] B. Helffer and S. Sjöstrand: Équation de Schrödinger avec champ magnétique et équation de Harper, in Schrödinger operators, H. Holden and A. Jensen, eds., Lecture Notes in Physics, vol. 345, Springer-Verlag, Berlin, 1989, pp. 118-197. MR 91g:35078

[Ho] L. Hörmander: The analysis of linear partial differential operators. I. Distribution theory and Fourier analysis, Second edition. Springer Study Edition, Springer-Verlag, Berlin, 1990. MR 91m:35001b

[JSBS] S. Jitomirskaya, H. Schulz-Baldes, and G. Stolz: Delocalization in random polymer models, Commun. Math. Phys. 233, 27-48 (2003).

[KLS] A. Klein, J. Lacroix, and A. Speis: Localization for the Anderson model on a strip with singular potentials, J. Funct. Anal. 94, 135-155 (1990). MR 92c:82060 
[Si] B. Simon: Schrödinger semi-groups, Bull. Amer. Math. Soc. (N.S.) 7, 447-526 (1982). MR 86b:81001a

[Tc] S. Tcheremchantsev: Dynamical analysis of Schrödinger operators with sparse potential, Comm. Math. Phys., to appear.

UMR 8524 CNRS, UFR de Mathématiques, Université de Lille 1, F-59655 Villeneuve D'Ascq Cédex, France

E-mail address: Jean-Marc.Bouclet@agat.univ-lille1.fr

UMR 8524 CNRS, UFR de Mathématiques, Université de Lille 1, F-59655 Villeneuve D'Ascq CÉdex, France

E-mail address: germinet@agat.univ-lille1.fr

Current address: Département de Mathématiques, Université de Cergy-Pontoise, Site de SaintMartin, 2 avenue Adolphe Chauvin, 95302 Cergy-Pontoise cedex, France

E-mail address: germinet@math.u-cergy.fr

Department of Mathematics, University of California Irvine, Irvine, California 926973875

E-mail address: aklein@uci.edu 